\title{
Tidal Perturbation and Active Galaxy Nuclei
}

\author{
Gene Byrd \\ Univ. of Alabama \\ Mauri Valtonen \\ Tuorla Observatory, Finland
}

\begin{abstract}
Theories of nuclear activity mechanisms in disk galaxies (such as Seyfert galaxies) range from intrinsic (e.g. bars) to external (tidal perturbation by passing companions). The Byrd et al. (1986, 1987) simulation survey determined the tidal perturbation necessary to induce nuclear activity. Observational surveys have raised doubt as to the tidal explanation of Seyfert-type activity in galaxies with some suggesting a weak or nonexistent correlation between tidal perturbation and Seyfert activity compared to a normal sample. The simulations show a several hundred million year delay in the appearance of activity after perturbation and a similar duration which can explain these null results. For double galaxies, we derive from the tidal hypothesis that: (1) There should be an excess of pairs where both are active over pairs with one active. (2) Nuclear activity will be preferentially excluded from pairs which are unequal and favorably distributed to pairs where the members are equal. (3) The size of pair members to create activity can be smaller if the pair members are equal in size than if they are unequal. (4) Pairs where one or both members are active should have a smaller velocity difference times separation than if both are normal. All the predictions are seen in observations of activity in Karachentsev's (1983) list of double galaxies. Optimally, statistical studies of nuclear activity and companions should include their sizes, separations, and radial velocities relative to the primary. The study by Dultzin-Hacyan et al. (1999) which finds an excess of large companions around Seyfert 2 galaxies is a step in the right direction.
\end{abstract}

\section{Tidal Perturbation Parameter}

We use Karachentsev's (1987) list of double galaxies as natural experiments to see if the predictions from simulations about tidally induced activity are valid. This list had non physical interlopers removed via radial velocity measurements of both members. Fig. 1 shows an example binary after Keel (1993). The disk radius, $A$, and diameter, $D$, for the larger, $L$, and smaller, $S$, members are indicated by subscripts. The present-day two dimensional projected separation 
on the sky is $b$. This is not usually the same as the three dimensional close approach distance, $B$, which probably occurred in the past.

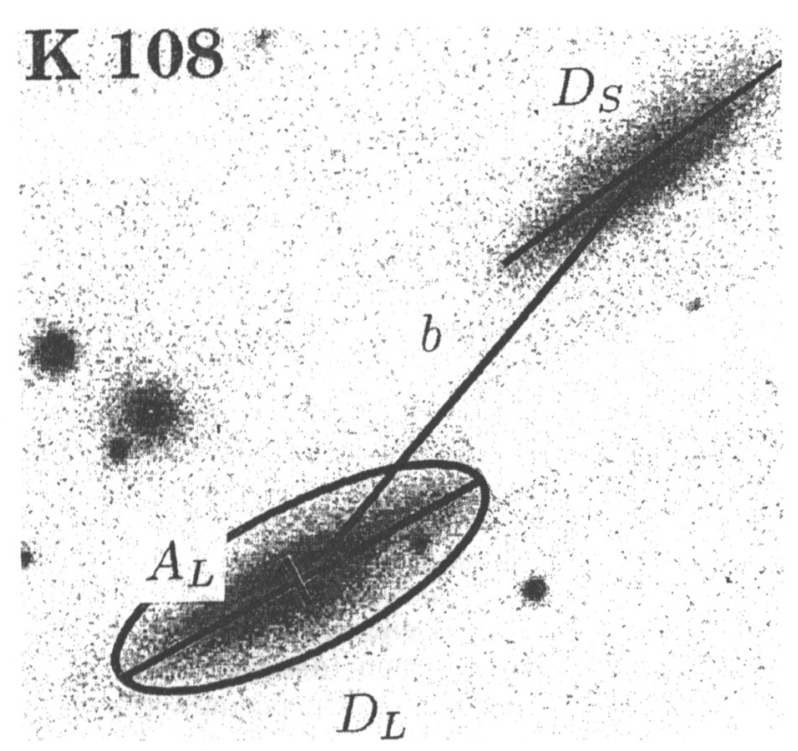

Figure 1. An example of a Karachentsev binary galaxy.

To compare to the double galaxies, we use a simulation survey in which Byrd et al. $(1986,1987)$ determined the critical level of tidal perturbation necessary to excite disk gas inflow into the disk center (within a radius of $1 / 20$ that of the disk) in amounts which observers cite as sufficient to fuel Seyfert activity. Perturbers of various masses, $\mathcal{M}_{S}$, encountered a galaxy, $\mathcal{M}_{L}$, containing a halo plus a 60,000 particle, self-gravitating, finite radius, flat rotation curve disk. Perturbers began in zero energy direct (same sense as disk "spin") or retrograde (opposite) orbits. Softening in the disk reproduced the Toomre stability parameter $Q>$ 1.0 over the entire disk. The simulation results are described with a single tidal parameter, the tidal acceleration/the gravitational acceleration toward the center at the perturbed galaxy's disk edge,

$$
P_{L}=\left(\frac{\mathcal{M}_{S} A_{L}}{B^{3}}\right) /\left(\frac{\mathcal{M}_{\mathcal{G}}}{A_{L}{ }^{2}}\right)=\frac{\left(\mathcal{M}_{S} / \mathcal{M}_{L}\right)}{\left(B / A_{L}\right)^{3}}
$$

Because Seyfert galaxies, aside from their nuclei, appear rather normal and because encounter statistics favor close approaches of smaller companions, the simulation survey included weak as well as strong tidal perturbation of disk galaxies. Close encounters with smaller mass perturbers and distant encounters with larger mass perturbers with the same value of $P_{L}$ produced similar inflows. 


\section{Disk Self-gravity, Orbit Sense, and Critical Perturbation}

Being conservative, no inelastic gas cloud collisions were considered in the disk by Byrd et al. $(1986,1987)$. Instead, inflow resulted from tidal perturbation and gravitational self-amplification which are the basic large scale inflow processes. If disk self-gravity is at all important, after perturbation, gravitational amplification occurs which propagates the disturbance into the nuclear regions. The halo to disk ratio, $H / D$, is the total orbital acceleration toward the center, $V_{e}^{2} / A$, divided by the gravitational contribution of the disk's mass to the acceleration toward the center. The smaller this ratio, the more self-gravitational amplification of disk disturbances will occur. The halo is usually presumed to be spherical but strictly speaking, it is a component of the galaxy which is more or less inert to gravitational amplification of disturbance because of its large velocity dispersion.

Because it is so poorly known, Byrd et al. tried many different values of $H / D$ in their simulations. For $H / D=1.0$ and direct (same sense as disk rotation) encounters, sufficient inflow to excite Seyfert activity (exc) is created when

$$
P_{\text {exc }, \text { dir }, 1 / 1} \geq 0.01 \text { versus a larger } P_{\text {exc,ret }, 1 / 1} \geq 0.0316
$$

for retrograde (opposite to disk spin) encounters. If the $H / D$ is increased to several times the galaxy disk contribution (designated by $3 / 1$ ), there is little difference between direct and retrograde in the encounters and

$$
P_{e x c, 3 / 1} \geq 0.1
$$

\section{Delay and Duration in Tidal Perturbation Activity}

In the simulations of a perturbed galaxy, after a delay $\left(d_{d e l}\right)$, the inflow rapidly rises to a peak then declines with sub-peaks over a duration $\left(d_{d u r}\right)$ after which it completely stops. The crossing time, $t$, is the disk radius divided by the disk edge speed, $V_{e}$. Typically, in the simulations, $d_{d u r} \approx d_{d e l}=d$. Typically, $d \approx 3 t$. For reasonable values,

$$
t=A / V_{e}=15 \mathrm{kpc} / 150 \mathrm{~km} \mathrm{~s}^{-1}=100 \times 10^{6} \mathrm{yr} \text { and } d=3 t=300 \times 10^{6} \mathrm{yr}
$$

We may see a disk galaxy which is more than sufficiently perturbed to create Seyfert activity but it will be several hundred million years before activity starts in the nucleus! The lack of Seyfert activity in merging or strongly interacting galaxies (Keel et al. 1985, and Bushouse 1986) is thus explained.

Studies of whether there is a difference in the number of nearby companions around active versus inactive galaxies have given contradictory results (e.g. Dahari 1984 and Keel et al. 1985 both find a difference whereas Laurikainen and Salo 1995 and De Robertis et al. 1998 do not). Taking the outward velocity of a companion to be $\sigma=200 \mathrm{~km} \mathrm{~s}^{-1}$ results in an outward movement during the delay and duration of $\sim 2 \sigma d=120 \mathrm{kpc}$, comparable to the search radii of the companion counts. Contradictory results can arise because the perturber can move far away perhaps not even being counted when the galaxy is in the delay 
and duration phases. Similarly, studies using an observational tidal perturbation like Eqn (1) but with encounter distance replaced by observed separation on the sky (Dahari 1984) would be affected by the change in the separation during delay and duration.

\section{Tidal Perturbation Properties for Double Galaxies}

According to Rubin et al. (1982) and Rubin (1983, Fig. 8), each spiral pair member's mass follows the relation

$$
\begin{gathered}
\mathcal{M} \propto A^{1.5} \\
\text { and } P_{S}=\frac{\mathcal{M}_{L} / \mathcal{M}_{S}}{\left(B / A_{S}\right)^{3}}=\frac{\left(2 A_{L}\right)^{1.5} /\left(2 A_{S}\right)^{1.5}}{\left(B / A_{S}\right)^{3}}=\frac{D_{L}^{1.5} D_{S}^{1.5}}{8 B^{3}}=P_{L}
\end{gathered}
$$

If one member is sufficiently perturbed then the other member of even unequal pairs is perturbed at the same time to the same level. We thus get our first observable prediction among doubles for the tidal hypothesis: (1) There should be an excess of pairs where both members are active (Both Mrk) and a deficiency of pairs with one member active (One Mrk). There should be a slight excess of Both Normal pairs.

We test this and other tidal predictions using a correlation of the double galaxy list with the observational survey of Markaryan (Mrk) or Seyfert active galaxies (Karachentsev 1983) as shown in Table 1. Karachentsev(1981) noted that there is an excess number of Both Mrk pairs. From Table 1, we see that there are $(299+50+12) 2=702$ pair members. Of these, $12 \times 2+50=74$ galaxies are active. If the association is random, we expect $(74 / 702)(74 / 702) 361=4$ pairs which are Both Mrk. The actual number is 12, an excess (as expected). Randomly, there should be 68 pairs with only one member active (One Mrk). The observed number is 50, a deficiency (as expected). Randomly, 289 "Both Normal" pairs are expected. The observed number is 299 , a slight excess. A $\chi^{2}$ test gives less than 0.001 probability that the Mrk activity is randomly distributed among the pair members. These numbers are functions of the mean delay and duration for the members of active pairs and thus of their sizes. Subject to the limitations of the small numbers, the results are consistent with the mean sizes of the large and small members of active pairs.

Recall that the mass of a disk galaxy,

$$
\mathcal{M} \propto V_{e}^{2} A \propto A^{1.5} \text { so that } t=A / V_{e} \propto A^{0.75}
$$

The time delay and duration of activity are thus approximately proportional to size. We thus expect central activity to appear more quickly in the galaxy with the smaller disk radius, $D$, in a sufficiently perturbed pair. Although the larger galaxy in a pair takes longer to show central activity, the activity lasts longer. If the pair members are equal in size, they will tend to be active at the same time. We thus have a new observable prediction: (2) Pairs where both members are active (Both Mrk) will tend to have members more equal in size than pairs where only one member is active. 
To test the hypothesis, in Fig. 2, we plot for each pair the larger member size on the ordinate and the smaller on the abscissa. In the plot of the One Mrk pairs, we expect and see a wide scattering of points above the 45 degree line. By definition, there are no points below a 45 degree line. Points for Both Mrk pairs cluster more closely to a 45 degree line. Via the Sigma Plot statistical package, we find that the points for the One Mrk pairs are 3.5 standard deviations above the Both Mrk points.

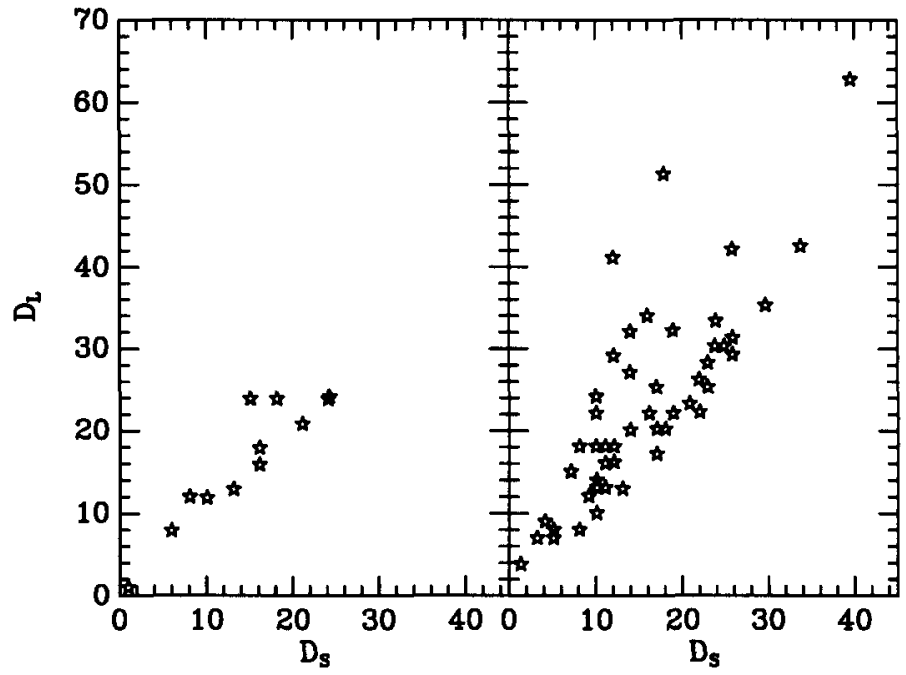

Figure 2. $\quad D_{L}$ vs $D_{S}$ for "Both Mrk" (Left) and "One Mrk" (Right).

Defining $f$ as a measure of the difference from equality of the pair members,

$$
\begin{gathered}
D_{a v}=\frac{\left(D_{L}+D_{S}\right)}{2} ; D_{L}=(1+f) D_{a v} ; \text { and } D_{S}=(1-f) D_{a v} \\
\text { and } P=\frac{\left(1-f^{2}\right)^{1.5} D_{a v}^{3}}{8 B^{3}}
\end{gathered}
$$

Designate as $D_{a v, 0}$ the average size for equal members sufficiently perturbed so as to be Both Mrk with $f$ of zero. For unequal Mrk pairs, use $D_{a v, f}$. Equation (7) becomes

$$
D_{a v, 0}=\left(1-f^{2}\right)^{0.5} D_{a v, f}
$$

For a given $P$ sufficient to create activity, $D_{a v}$ does not have to be as large if $f=0$ compared to an unequal pair. We thus get a third observational prediction, (3) The average size of the pair members can be smaller to create activity if the pair members are equal in size (Both Mrk) than if they are unequal (One Mrk or Both Normal). 
Karachentsev(1981) noted that the pairs in which one or both members are Mrk have both components smaller than the normal pairs. Table 1 shows the mean sizes of the larger and the smaller members of the Both Normal, One Mrk, and Both Mrk pairs. The difference for the Both Mrk pairs compared to the normal pairs is over two standard deviations between the average sizes of the members. There is even a significant difference between the Both Mrk and One Mrk samples.

To trigger activity, we expect that the encounter orbit for One or Both Mrk pairs should have closer approach distances $B$, than those for Both Normal pairs. Tidal orbital modification may also have occurred during the close approach (e.g. Valtaoja 1990) so as to reduce the close encounter speed, $V_{\text {close }}$. In terms of observable quantities, we expect the current radial velocity difference, $v$, times the separation on the sky, $b$, to be smaller. This product is $b v=\left|\vec{B} \times \vec{V}_{\text {close }}\right|_{y}$, the $y$ component of the angular momentum which is conserved after the close approach. Thus, $B \propto b v / V_{\text {close }}$.

Thus, we get a fourth prediction: (4) bv $\propto B$ should be smaller for One or Both Mrk pairs compared to Both Normal pairs. Karachentsev(1981) noted that, on the average, pairs with one or both Mrk members had a smaller separation than Both Normal members. He also noted that the relative velocities are smaller. Examining Table 2, there are about three standard deviations between the Both Normal and Mrk pairs' average velocities and over two standard deviations between the separations. Multiplying these two, we see the estimated close approach distance of a Mrk active pair is definitely less than that of a normal pair.

Acknowledgments. This work was supported by the Academy of Finland. GB expresses gratitude for the hospitality of Tuorla Observatory in Finland during a winter/spring 1999 visit and for David Standifer's scanning of illustrations.

\section{References}

Bushouse, H. A. 1986, AJ, 91, 255

Byrd, G. G., Sundelius, B. and Valtonen, M. 1987, A\&A, 171, 16

Byrd, G. G., Valtonen, M., Sundelius, B., and Valtaoja, L. 1986 A\&A, 166, 75

Dahari, O., 1984, AJ, 89, 966

Dahari, O., 1985, AJ, 57, 643

DeRobertis, M. M., Yee, H. K. C., and Hayhoe, K. 1998, ApJ, 496, 93

Dultzin-Hacyan, D., Krongold, Y., Fuentes-Guridi, I., and Marziani, P. 1999, ApJL, 513, L111

Karachentsev, I. D. 1981, Soviet Astron. Lett. (Tr. Pisma Astr. Zh.), 7,4 1

Karachentsev I. D. Binary Galaxies. Nauka, Moscow, 1987, (in Russian)

Keel, W. C., Kennicutt, R. Jr., Hummel, E., van der Hulst, J. M. 1985, AJ, 90, 708.

Laurikainen, E. and Salo, H. 1995, A\&A, 293, 683

Rubin, V. C. 1983, In IAU Symposium \#100, Internal Kinematics and Dynamics of Spiral Galaxies. D. Reidel, Publishers. Editor, E. Athanassoula, p. 3. 
Rubin, V. C., Ford, Jr. W. K., Thonnard, N. and Burstein, D. 1982, ApJ, 261, 439

Valtaoja, L. 1990 A\&A, 228, 37

Table 1. NORMAL/MRK DOUBLES-NUMBERS AND MEAN SIZES

\begin{tabular}{|c|c|c|c|}
\hline Parameter & Both Normal & One Mrk & Both Mrk \\
\hline Observed Number $(O)$ & 299 & 50 & 12 \\
\hline Random Distrib. (R) & 289 & 68 & \\
\hline$(O-R)^{2} / R$ & 0.3 & 4.8 & 16 \\
\hline $\begin{array}{c}\chi^{2}=21 \\
\operatorname{Prob}_{O=R}<0.001\end{array}$ & & & \\
\hline$\left\langle D_{L}\right\rangle \mathrm{kpc}$ & $26.6 \pm 0.4$ & $23.8 \pm 1.3$ & $16.4 \pm$ \\
\hline$\left\langle D_{S}\right\rangle \mathrm{kpc}$ & $18.6 \pm 0.35$ & $16.5 \pm 1$ & $14.4 \pm 2$ \\
\hline$<\frac{D_{L}+D_{S}}{2}>\mathrm{kpc}$ & $\begin{array}{r}22.6 \pm 0.4 \\
7 ?+18\end{array}$ & $20 \pm 0.8$ & $15.4 \pm 1.8$ \\
\hline
\end{tabular}

Table 2. NORMAL/MRK DOUBLES-ENCOUNTER DISTANCES

\begin{tabular}{crrr}
\hline Parameter & Both Normal & One or Both Mrk & $N-M r k$ \\
$\left\langle v>\mathrm{km} \mathrm{s}^{-1}\right.$ & $128 \pm 4$ & $95 \pm 9$ & $33 \pm 9.9$ \\
$<b>\mathrm{kpc}$ & $40 \pm 2$ & $29 \pm 4$ & $11 \pm 4.5$ \\
$B \propto b v$ & $5120 \pm 302$ & $2755 \pm 437$ & $2365 \pm 531$ \\
\hline
\end{tabular}

